\title{
Do Small-Mass Neutrinos Participate in Gauge Transformations?
}

\author{
Y. S. Kim, ${ }^{1}$ G. Q. Maguire Jr., ${ }^{2}$ and M. E. $\mathrm{Noz}^{3}$ \\ ${ }^{1}$ Center for Fundamental Physics, University of Maryland, College Park, MD 20742, USA \\ ${ }^{2}$ School of Information Technology, KTH Royal Institute of Technology, 16440 Stockholm, Sweden \\ ${ }^{3}$ Department of Radiology, New York University, New York, NY 10016, USA
}

Correspondence should be addressed to M. E. Noz; marilyne.noz@gmail.com

Received 31 March 2016; Revised 25 May 2016; Accepted 12 June 2016

Academic Editor: Theocharis Kosmas

Copyright ( $\odot 2016$ Y. S. Kim et al. This is an open access article distributed under the Creative Commons Attribution License, which permits unrestricted use, distribution, and reproduction in any medium, provided the original work is properly cited. The publication of this article was funded by SCOAP

\begin{abstract}
Neutrino oscillation experiments presently suggest that neutrinos have a small but finite mass. If neutrinos have mass, there should be a Lorentz frame in which they can be brought to rest. This paper discusses how Wigner's little groups can be used to distinguish between massive and massless particles. We derive a representation of the $S L(2, c)$ group which separates out the two sets of spinors: one set is gauge dependent and the other set is gauge invariant and represents polarized neutrinos. We show that a similar calculation can be done for the Dirac equation. In the large-momentum/zero-mass limit, the Dirac spinors can be separated into large and small components. The large components are gauge invariant, while the small components are not. These small components represent spin-1/2 non-zero-mass particles. If we renormalize the large components, these gauge invariant spinors represent the polarization of neutrinos. Massive neutrinos cannot be invariant under gauge transformations.
\end{abstract}

\section{Introduction}

Whether or not neutrinos have mass and the consequences of this relative to the Standard Model and lepton number are the subject of much theoretical speculation $[1,2]$, as well as cosmological [3-5], nuclear reactor [6,7], and high energy experimentation [8-11]. Neutrinos are fast becoming an important component of the search for dark matter and dark radiation $[12,13]$. Their importance within the Standard Model is reflected in the fact that they are the only particles which seem to exist with only one direction of chirality; that is, only left-handed neutrinos have been confirmed to exist thus far. It was speculated some time ago that neutrinos in constant electric and magnetic fields would acquire a small mass and that right-handed neutrinos would be trapped within the interaction field [14]. Additionally there are several physical problems which right-handed neutrinos might help solve [15-17]. Solving generalized electroweak models using left- and right-handed neutrinos has also been discussed [18]. Today right-handed neutrinos which do not participate in weak interactions are called "sterile" neutrinos [19]. A comprehension discussion of the place of neutrinos in the present scheme of particle physics has been given by Drewes [12].

In this paper, we use representations of the Lorentz group to understand the physical implications of neutrinos having mass. In Section 2, two-by-two representations of the Lorentz group are presented. In Section 3, the internal symmetries of massive and massless particles are derived. A representation of the $S L(2, c)$ group, which separates out the two sets of spinors contained therein, is presented in Section 4. One set of spinors is gauge dependent and represents massive particles. The other is gauge invariant and represents polarized neutrinos. In Section 5, we show how, in the large-momentum/zero-mass limit, the Dirac spinors can be separated into two components, one of which can represent a spin-1/2 non-zero-mass particle. The question of gauge invariance is then discussed. In Section 6, we discuss the zero-mass limit and gauge invariance in the Lorentz transformation framework. Some concluding remarks are made in Section 7. 


\section{Representations of the Lorentz Group}

The Lorentz group starts with a group of four-by-four matrices performing Lorentz transformations on the fourdimensional Minkowski space of $(t, z, x, y)$ which leaves the quantity $\left(t^{2}-z^{2}-x^{2}-y^{2}\right)$ invariant. Since there are three generators of rotations and three boost generators, the Lorentz group is a six-parameter group.

Einstein observed that the Lorentz group is also applicable to the four-dimensional energy and momentum space of $\left(E, p_{z}, p_{x}, p_{y}\right)$. He derived the Lorentz-covariant energymomentum relation commonly known as $E=m c^{2}$. As this transformation leaves $\left(E^{2}-p_{z}^{2}-p_{x}^{2}-p_{y}^{2}\right)$ invariant, the particle mass is a Lorentz invariant quantity.

In his 1939 paper [20], Wigner studied the symmetry properties of free particles by using operators which commute with the specified four-momentum of the particle. His "little groups" were defined to be those transformations that do not change this four-momentum. For massive particles, the little group is isomorphic to $O(3)$; indeed the $O(3)$-like little group's kinematics is well understood. Massless particles are isomorphic to the Euclidean group commonly known as $E(2)$. Wigner noted that the $E(2)$-like subgroup of $S L(2, c)$ is isomorphic to the Lorentz group of transformations [21], but the kinematics of this group is not as well established as that of the $O(3)$-like little group as there is no Lorentz frame in which a massless particle is at rest.

It is possible to construct the Lie algebra of the Lorentz group from the three Pauli spin matrices [22-25] as

$$
\begin{aligned}
J_{i} & =\frac{1}{2} \sigma_{i}, \\
K_{i} & =\frac{i}{2} \sigma_{i} .
\end{aligned}
$$

These two-by-two matrices satisfy the following set of commutation relations:

$$
\begin{aligned}
{\left[J_{i}, J_{j}\right] } & =i \epsilon_{i j k} J_{k}, \\
{\left[J_{i}, K_{j}\right] } & =i \epsilon_{i j k} K_{k}, \\
{\left[K_{i}, K_{j}\right] } & =-i \epsilon_{i j k} J_{k},
\end{aligned}
$$

where the generators $J_{i}$ represent rotations and the generators $K_{i}$ represent boosts. There are six generators of the Lorentz group which satisfy the three sets of commutation relations given in (2). The Lie algebra of the Lorentz group consists of these sets of commutation relations.

These commutation relations are invariant under Hermitian conjugation; however, while the rotation generators are Hermitian, the boost generators are anti-Hermitian:

$$
\begin{aligned}
J_{i}^{\dagger} & =J_{i}, \\
\text { while } K_{i}^{\dagger} & =-K_{i} .
\end{aligned}
$$

Thus, it is possible to construct two representations of the Lorentz group, one with $K_{i}$ and the other with $-K_{i}$. For this purpose, we will use the notation $[24,26,27]$

$$
\dot{K}_{i}=-K_{i}
$$

To demonstrate that this set of generators do perform Lorentz transformations, let us consider a point $X$ in fourdimensional space such as the Minkowskian four-vector $(t, z, x, y)$. A Hermitian matrix of the form

$$
X=\left(\begin{array}{cc}
t+z & x-i y \\
x+i y & t-z
\end{array}\right),
$$

with determinant

$$
t^{2}-z^{2}-x^{2}-y^{2}
$$

can be written where all the components of $X$ are real. Indeed, every Hermitian matrix can be written this way with real components. Consider next a matrix of the form

$$
G=\left(\begin{array}{ll}
\alpha & \beta \\
\gamma & \delta
\end{array}\right),
$$

with four complex matrix elements, thus eight real parameters, and require that the determinant be equal to one. If

$$
G^{\dagger}=\left(\begin{array}{ll}
\alpha^{*} & \gamma^{*} \\
\beta^{*} & \delta^{*}
\end{array}\right)
$$

is the Hermitian conjugate of $G$, then

$$
X^{\prime}=G X G^{\dagger}
$$

defines a linear transformation with real coefficients such that the determinant of $X^{\prime}$ is equal to the determinant of $X$. This constitutes a real Lorentz transformation. The transformation of (9) can be explicitly written as

$$
\begin{aligned}
& \left(\begin{array}{cc}
t^{\prime}+z^{\prime} & x^{\prime}-i y^{\prime} \\
x^{\prime}+i y^{\prime} & t^{\prime}-z^{\prime}
\end{array}\right) \\
& =\left(\begin{array}{ll}
\alpha & \beta \\
\gamma & \delta
\end{array}\right)\left(\begin{array}{cc}
t+z & x-i y \\
x+i y & t-z
\end{array}\right)\left(\begin{array}{ll}
\alpha^{*} & \gamma^{*} \\
\beta^{*} & \delta^{*}
\end{array}\right) .
\end{aligned}
$$

It is important to note that the transformation of (9) is not a similarity transformation. In the $S L(2, c)$ regime, not all the matrices are Hermitian [25]. Moreover, since the determinants of $G$ and $G^{\dagger}$ are one, the determinant of $G G^{\dagger}$ is also one. As

$$
\operatorname{Tr}\left(G G^{\dagger}\right)=\left(\alpha \alpha^{*}+\beta \beta^{*}+\gamma \gamma^{*}+\delta \delta^{*}\right) \geq 1,
$$

(9) is a proper Lorentz transformation [25, 28, 29].

Since the determinant of $G$ is fixed and is equal to one, there are six independent parameters. This six-parameter group is commonly called $S L(2, c)$. As the Lorentz group has six generators, this two-by-two matrix can serve as a representation of the Lorentz group.

Likewise, the two-by-two matrix for the four-momentum of the particle takes the form

$$
P=\left(\begin{array}{cc}
p_{0}+p_{z} & p_{x}-i p_{y} \\
p_{x}+i p_{y} & p_{0}-p_{z}
\end{array}\right)
$$


with $p_{0}=\sqrt{m^{2}+p_{z}^{2}+p_{x}^{2}+p_{2}^{2}}$. The transformation of this matrix takes the same form as that for space-time given in (9) and (10). The determinant of this matrix is $m^{2}$ and remains invariant under Lorentz transformations. The explicit form of the transformation is

$$
\begin{aligned}
P^{\prime} & =G P G^{\dagger}=\left(\begin{array}{cc}
p_{0}^{\prime}+p_{z}^{\prime} & p_{x}^{\prime}-i p_{y}^{\prime} \\
p_{x}^{\prime}+i p_{y}^{\prime} & p_{0}^{\prime}-p_{z}^{\prime}
\end{array}\right) \\
& =\left(\begin{array}{ll}
\alpha & \beta \\
\gamma & \delta
\end{array}\right)\left(\begin{array}{ll}
p_{0}+p_{z} & p_{x}-i p_{y} \\
p_{x}+i p_{y} & p_{0}-p_{z}
\end{array}\right)\left(\begin{array}{ll}
\alpha^{*} & \gamma^{*} \\
\beta^{*} & \delta^{*}
\end{array}\right) .
\end{aligned}
$$

It is this Lorentz invariant mass that is important for discussing neutrino oscillation. In the next section, the internal symmetry of particles will be discussed using Wigner's little groups.

\section{Internal Symmetries of Massive and Massless Particles}

When special relativity was formulated, the main focus was point particles, without internal space-time structures. How these particles look to moving observers can be studied using Wigner's little groups [20] where the subgroup of the Lorentz group whose transformations leave the particle momentum invariant are considered. However, the little groups can transform the internal space-time structure of the particles. Since the particle momentum is fixed and remains invariant, it is possible to consider that the particle momentum is along the $z$ direction.

This momentum is thus invariant under rotations around the $z$-axis. In addition, these rotations commute with the Lorentz boost along the $z$-axis because, according to the Lie algebra of (2),

$$
\left[J_{3}, K_{3}\right]=0 \text {. }
$$

In Section 2, it was shown that the Lorentz transformation of the four-momentum can be represented by two-by-two matrices and an explicit form for this transformation was given. If the particle moves along the $z$ direction, the fourmomentum matrix becomes

$$
P=\left(\begin{array}{cc}
E+p & 0 \\
0 & E-p
\end{array}\right),
$$

where $E$ and $p$ are the energy and the magnitude of momentum, respectively.

Let $W$ be a subset of matrices which leaves the fourmomentum invariant; then we can write

$$
P=W P W^{\dagger} \text {. }
$$

These matrices constitute Wigner's little groups dictating the internal space-time symmetry of the particle.

If the particle is massive, it can be brought to the system where it is at rest with $p=0$. The four-momentum matrix is then proportional to

$$
P=\left(\begin{array}{ll}
1 & 0 \\
0 & 1
\end{array}\right) .
$$

Since the momentum matrix is proportional to the unit matrix, the $W$ matrix forms a unitary subset of the $G$ matrices and is Hermitian. The corresponding little group is the SU(2) subgroup of the Lorentz group. It is sufficient to consider rotations around the $y$-axis, as rotations around the $z$-axis do not change the momentum. Thus the rotation matrix

$$
R(\theta)=\left(\begin{array}{cc}
\cos \left(\frac{\theta}{2}\right) & -\sin \left(\frac{\theta}{2}\right) \\
\sin \left(\frac{\theta}{2}\right) & \cos \left(\frac{\theta}{2}\right)
\end{array}\right)
$$

can be used. This forms a representation of Wigner's $O(3)$ like little group for massive particles which describes the spin orientation of the particle in the rest frame.

For the massless particle, $E=p$. Thus the fourmomentum matrix is proportional to

$$
P=\left(\begin{array}{ll}
1 & 0 \\
0 & 0
\end{array}\right),
$$

and the Wigner matrix is necessarily triangular and should take the form

$$
T(\gamma)=\left(\begin{array}{cc}
1 & -\gamma \\
0 & 1
\end{array}\right) .
$$

This matrix cannot be diagonalized. Its inverse and Hermitian conjugate are

$$
\begin{aligned}
& T^{-}(\gamma)=\left(\begin{array}{ll}
1 & \gamma \\
0 & 1
\end{array}\right), \\
& T^{\dagger}(\gamma)=\left(\begin{array}{ll}
1 & 0 \\
-\gamma & 1
\end{array}\right),
\end{aligned}
$$

respectively. Since the inverse is not the same as the Hermitian conjugate, $T$ is not a Hermitian matrix. In order to preserve the Lorentz properties of the boosted fourmomentum, $\gamma$ must be real.

To understand this better, consider that as the $O(3)$ group is contracted into the Euclidean group $(E(2))$ group, one can think of $E(2)$ as a plane tangent to the North Pole. Since $E(2)$ consists of two translation operators and a rotation operator, the rotation around the $z$-axis remains unchanged as the radius becomes large and rotations around the $x$ - and $y$-axes become translations in the $x$ and $-y$ directions, respectively, within the tangent plane. For a massless particle, the $E(2)$ like little group bears the same relation to the $E(2)$ group as the $O(3)$-little group does to $O(3)$ for a massive particle. Thus (20) is the representation of Wigner's $E(2)$-like little group $[21,27,30]$ for massless particles. It is now possible to apply this formalism to spin-1/2 particles by considering the $S L(2, c)$ representation of the Lorentz group.

\section{4. $S L(2, c)$ and Spinors}

In the case of $S L(2, c)$, or spin- $1 / 2$ particles, it is necessary to consider both signs of the boost generators $K_{i}$. In Section 2, 
we considered that $S L(2, c)$ consists of nonsingular two-bytwo matrices which have the form defined in (7). This matrix is applicable to spinors that have the following form:

$$
\begin{aligned}
& U=\left(\begin{array}{l}
1 \\
0
\end{array}\right), \\
& V=\left(\begin{array}{l}
0 \\
1
\end{array}\right),
\end{aligned}
$$

for spin-up and spin-down states, respectively.

Among the subgroups of $S L(2, c)$, there are $E(2)$-like little groups which correspond to massless particles. If we consider a massless particle moving along the $z$ direction, then the little group is generated by $J_{3}, N_{1}$, and $N_{2}$, where

$$
\begin{aligned}
& N_{1}=K_{1}-J_{2}, \\
& N_{2}=K_{2}+J_{1}, \\
& J_{3}=\left(\frac{1}{2}\right) \sigma_{3} .
\end{aligned}
$$

As usual, $J_{3}$ is the generator of rotations and $N_{i}$ generate translation-like transformations where

$$
D(u, v)=D(u, 0) D(0, v)=D(0, v) D(u, 0) .
$$

As these $N$ operators have been shown to be the generators of gauge transformations in the case of the photon [21], they will be referred to as the gauge transformation in the $S L(2, c)$ regime $[20,31-33]$. Their role with respect to massless particles of spin-1/2 will now be discussed [25].

For massless spin-1/2 particles, $J_{i}$ are still the generators of rotations. However, because of the sign change allowed for $K_{i}$, it is necessary to have two sets of $N_{i}$ operators designated as $N_{i}^{(+)}$and $N_{i}^{(-)}$, where, as defined in (23), $N_{i}^{(+)}$have the explicit form

$$
\begin{aligned}
& N_{1}^{(+)}=\left(\begin{array}{ll}
0 & i \\
0 & 0
\end{array}\right), \\
& N_{2}^{(+)}=\left(\begin{array}{ll}
0 & 1 \\
0 & 0
\end{array}\right) .
\end{aligned}
$$

The Hermitian conjugates of the above $N_{i}^{(+)}$provide $N_{1}^{(-)}$and $N_{2}^{(-)}$. Thus, there are two sets of boost generators involved.

The transformation matrices defined in (24) can then be written as [34]

$$
\begin{aligned}
D^{(+)}(u, v) & =\exp \left(-i\left[u N_{1}^{[+]}+v N_{2}^{[+]}\right]\right) \\
& =\left(\begin{array}{cc}
1 & u-i v \\
0 & 1
\end{array}\right), \\
D^{(-)}(u, v) & =\exp \left(-i\left[u N_{1}^{[-]}+v N_{2}^{[-]}\right]\right) \\
& =\left(\begin{array}{rr}
1 & 0 \\
-u-i v & 1
\end{array}\right) .
\end{aligned}
$$

Since there are two sets of spinors in $S L(2, c)$, the spinors whose boosts are generated by $K_{i}=i / 2 \sigma$ will be written as $\alpha$ for spin in the positive direction and $\beta$ for spin in the negative direction. For the boosts generated by $K_{i}=-i / 2 \sigma$, we will use $\dot{\alpha}$ and $\dot{\beta}$. These spinors are gauge invariant in the sense that

$$
\begin{aligned}
& D^{(+)}(u, v) \alpha=\alpha, \\
& D^{(-)}(u, v) \dot{\beta}=\dot{\beta} .
\end{aligned}
$$

However, if we carry out the explicit multiplication, these spinors are gauge dependent in the sense that

$$
\begin{aligned}
& D^{(+)}(u, v) \beta=\beta+(u-i v) \alpha, \\
& D^{(-)}(u, v) \dot{\alpha}=\dot{\alpha}-(u+i v) \dot{\beta} .
\end{aligned}
$$

The gauge invariant spinors of (27) appear as polarized neutrinos [21, 30, 35].

Let us examine further the gauge dependent spinors of (28). To accomplish this, we construct unit vectors in Minkowskian space by taking the direct product of two $S L(2, c)$ spinors:

$$
\begin{aligned}
-\alpha \dot{\alpha} & =(1, i, 0,0), \\
\beta \dot{\beta} & =(1,-i, 0,0), \\
\alpha \dot{\beta} & =(0,0,1,1), \\
\beta \dot{\alpha} & =(0,0,1,-1) .
\end{aligned}
$$

This combines two half integer spins into integer spins. To make $D(u, v)$ consistent with (29), it is necessary to choose

$$
D(u, v)=D^{(+)}(u, v) D^{(-)}(u, v)
$$

where $D^{(+)}$and $D^{(-)}$apply to the first and second spinors of (29), respectively. Since the plane wave photon four-potential does not depend on $\beta \dot{\alpha}$ because of the Lorentz condition [21, $30,31,34,35]$, we have

$$
\begin{aligned}
D(u, v)(-\alpha \dot{\alpha}) & =-\alpha \dot{\alpha}+(u+i v) \alpha \dot{\beta}, \\
D(u, v)(\beta \dot{\beta}) & =\beta \dot{\beta}+(u-i v) \alpha \dot{\beta}, \\
D(u, v) \alpha \dot{\beta} & =\alpha \dot{\beta} .
\end{aligned}
$$

The first two equations in (31) correspond to gauge transformations of the photon polarization vectors. The third equation corresponds to the effect of the $D$ transformation of the four-momentum. This shows that $D(u, v)$ is an element of the little group. We look next at how we can apply this analysis to Dirac spinors.

\section{Dirac Spinors and Massless Particles}

The Dirac equation is applicable to massive particles. Here we will consider the massless particle as the limiting case of the massive particle by considering the large-momentum/zeromass limit of the Dirac spinors. 
Starting with the spin operators defined in (1), a boost along the $z$ direction will take the form

$$
J_{i}^{\prime}=B(P) J_{i} B^{-1}(P) .
$$

This is a similarity transformation. Here the boost matrix is given by

$$
B(P)=\left(\begin{array}{cc}
e^{\eta / 2} & 0 \\
0 & e^{-\eta / 2}
\end{array}\right)
$$

where

$$
e^{\eta / 2}=\left(\frac{E+P}{E-P}\right)
$$

In the large-momentum or large-mass limit for a massive particle we obtain

$$
e^{\eta} \longrightarrow \frac{2 E}{M}
$$

Using the similarity transformation of $(32), J_{3}$ is invariant, but $J_{1}$ and $J_{2}$ take the form

$$
\begin{aligned}
& J_{1}^{\prime}=\left(\begin{array}{cc}
0 & \frac{1}{2} e^{\eta} \\
\frac{1}{2} e^{-\eta} & 0
\end{array}\right), \\
& J_{2}^{\prime}=\left(\begin{array}{cc}
0 & -\frac{i}{2} e^{\eta} \\
\frac{i}{2} e^{-\eta} & 0
\end{array}\right) .
\end{aligned}
$$

In the large-momentum or large-mass limit for a massive particle, we can obtain the $N_{i}$ matrices of (23) as

$$
\begin{aligned}
& N_{1}=-\frac{M}{E} J_{2}^{\prime}, \\
& N_{2}=\frac{E}{M} J_{1}^{\prime} .
\end{aligned}
$$

Remembering that we have to consider both signs of the boost generators, the generators of $\operatorname{SL}(2, c)$ can take the form

$$
\begin{aligned}
J_{i} & =\left(\begin{array}{cc}
\left(\frac{1}{2}\right) \sigma_{i} & 0 \\
0 & \left(\frac{1}{2}\right) \sigma_{i}
\end{array}\right), \\
K_{i} & =\left(\begin{array}{cc}
\left(\frac{i}{2}\right) \sigma_{i} & 0 \\
0 & \left(-\frac{i}{2}\right) \sigma_{i}
\end{array}\right)
\end{aligned}
$$

which is applicable to Dirac wave functions in the Weyl representation $[14,24]$. Using the gauge transformation matrices from (26), we can write

$$
D(u, v)=\left(\begin{array}{cc}
D^{(+)}(u, v) & 0 \\
0 & D^{(-)}(u, v)
\end{array}\right)
$$

This matrix is applicable to the Dirac spinors. To evaluate the result of applying the $D$ matrix from (39), we first look at the eigenspinors given in (22) applied to a massive Dirac particle that is at rest. Thus we have

$$
\begin{aligned}
& U(0)=\left(\begin{array}{c}
\alpha \\
\pm \dot{\alpha}
\end{array}\right), \\
& V(0)=\left(\begin{array}{c} 
\pm \beta \\
\dot{\beta}
\end{array}\right),
\end{aligned}
$$

where the positive and negative energy states are denoted by the + and - signs, respectively. If these spinors are boosted along the $z$-axis using the operator generated by $K_{3}$, then

$$
\begin{aligned}
& U(P)=\left(\begin{array}{c}
e^{(+\eta / 2)} \alpha \\
\pm e^{(-\eta / 2)} \dot{\alpha}
\end{array}\right), \\
& V(P)=\left(\begin{array}{c} 
\pm e^{(-\eta / 2)} \beta \\
e^{(+\eta / 2)} \dot{\beta}
\end{array}\right) .
\end{aligned}
$$

In the large-momentum/zero-mass limit, the large components, $e^{(+\eta / 2)}$, are, according to (27), gauge invariant, while the small components, according to (28), are gauge dependent. This again shows that non-zero-mass, spin-1/2 particles are not invariant under gauge transformations. Furthermore, in this limit, the spinors of (41) can be renormalized as

$$
\begin{aligned}
& U(P)=\left(\begin{array}{l}
\alpha \\
0
\end{array}\right), \\
& V(P)=\left(\begin{array}{l}
0 \\
\dot{\beta}
\end{array}\right) .
\end{aligned}
$$

It is clear that the $D$ transformation leaves these spinors invariant. It is this invariance, as shown before, that is responsible for the polarization of neutrinos [30, 35].

Additionally, one could interpret the results of (41) in terms of $E(2)$ translations on free Weyl neutrino states. In this case, the gauge invariant transformations leave the lefthanded neutrino invariant but translate the right-handed neutrino into a linear combination of left-handed and righthanded neutrinos $[14,30]$. These coupled states could have implications requiring that in a constant electric and magnetic field neutrinos should acquire a small effective mass [14].

\section{Neutrino Mass and Lorentz Transformations}

In Section 3, we introduced the fact that Wigner [20] proposed that his "little groups" be defined as those Lorentz transformations that do not change the four-momentum of the free particle. Because there is no Lorentz frame in which a massless particle is at rest, we had to consider a momentum four-vector of the form given in (19). From this we were able to write down the transformation matrix given in (20) which 
left the four-momentum invariant. In this section, we begin with a massive particle with fixed energy $E$. Then we can write the Lorentz boost along the $z$ direction as

$$
\begin{gathered}
z \longrightarrow(\cosh \xi) z+(\sinh \xi) t, \\
t \longrightarrow(\sinh \xi) z+(\cosh \xi) t .
\end{gathered}
$$

As we saw in Section 5 , the limiting case of $e^{\xi}$ is given in (35) for the large-momentum limit. Within the framework of Lorentz transformations, $E$ can become large and thus $\xi$ can also become large. This has been discussed in the literature [24].

In addition, $\xi$ can become large when the mass becomes very small. This cannot be achieved by Lorentz boosts, because the mass is a Lorentz-invariant quantity. With this point in mind, we can consider what happens when the mass is varied but the energy is held fixed. We can write the energymomentum four-vector as

$$
E(0,0, \cos \chi, 1) \text {. }
$$

Then the mass becomes

$$
M=E \sqrt{1-\cos ^{2} \chi}=E \sin \chi
$$

Hence, the mass can be increased by increasing $\chi$ from zero [36].

While the four-by-four matrix which makes the transformation of (43) is

$$
\left(\begin{array}{cccc}
1 & 0 & 0 & 0 \\
0 & 1 & 0 & 0 \\
0 & 0 & \cosh \xi & \sinh \xi \\
0 & 0 & \sinh \xi & \cosh \xi
\end{array}\right),
$$

its two-by-two equivalent to the spinor is

$$
\left(\begin{array}{cc}
e^{\xi / 2} & 0 \\
0 & e^{-\xi / 2}
\end{array}\right)
$$

as seen in (33) [36]. The two-by-two matrix corresponding to a rotation around the $y$-axis is

$$
\left(\begin{array}{cc}
\cos \left(\frac{\theta}{2}\right) & -\sin \left(\frac{\theta}{2}\right) \\
\sin \left(\frac{\theta}{2}\right) & \cos \left(\frac{\theta}{2}\right)
\end{array}\right)
$$

Thus we can now perform the Lorentz boost by making a similarity transformation:

$$
\left(\begin{array}{cc}
e^{\xi / 2} & 0 \\
0 & e^{-\xi / 2}
\end{array}\right)\left(\begin{array}{cc}
\cos \left(\frac{\theta}{2}\right) & -\sin \left(\frac{\theta}{2}\right) \\
\sin \left(\frac{\theta}{2}\right) & \cos \left(\frac{\theta}{2}\right)
\end{array}\right)\left(\begin{array}{cc}
e^{-\xi / 2} & 0 \\
0 & e^{\xi / 2}
\end{array}\right)
$$

which becomes

$$
\left(\begin{array}{cc}
\cos \left(\frac{\theta}{2}\right) & -e^{\xi} \sin \left(\frac{\theta}{2}\right) \\
e^{-\xi} \sin \left(\frac{\theta}{2}\right) & \cos \left(\frac{\theta}{2}\right)
\end{array}\right)
$$

For this matrix to remain finite in the large $\xi$ limit, we can let $e^{\xi} \sin \theta=\gamma$. For $\gamma$ to remain finite as $\xi$ increases, $\theta$ must approach zero. Then, in the limiting case, the matrix given in (50) becomes

$$
\left(\begin{array}{cc}
1 & -\gamma \\
0 & 1
\end{array}\right) .
$$

It has been shown that the $\gamma$ parameter performs gauge transformations on the photon case and its equivalent transformation on massless neutrinos [24, 30, 35]. If the neutrino indeed has mass, then we should observe neutrinos participating in gauge transformations.

\section{Concluding Remarks}

As there is currently much interest in massive neutrinos, it would be interesting to see if there was indeed a Lorentz frame in which neutrinos could be brought to rest. Additionally, it would be useful to understand if neutrinos participate in gauge transformations. The issue of whether or not the neutrino is a Dirac particle as opposed to a Majorana particle will be settled only if lepton number violation is observed. Furthermore, if right-handed neutrinos could be found separated from left-handed neutrinos, and if these righthanded neutrinos did not participate in weak interactions, this would have implications for physics beyond the Standard Model.

\section{Competing Interests}

The authors declare that they have no competing interests.

\section{References}

[1] D. K. Papoulias and T. S. Kosmas, "Exotic lepton flavour violating processes in the presence of nuclei," Journal of Physics: Conference Series, vol. 410, no. 1, Article ID 012123, 2013.

[2] D. N. Dinh, S. T. Petcov, N. Sasao, M. Tanaka, and M. Yoshimura, "Observables in neutrino mass spectroscopy using atoms," Physics Letters, Section B: Nuclear, Elementary Particle and High-Energy Physics, vol. 719, no. 1-3, pp. 154-163, 2013.

[3] L. Miramonti and V. Antonelli, "Advancements in solar neutrino physics," International Journal of Modern Physics E, vol. 22, no. 5, Article ID 1330009, 2013.

[4] A. Higuera, A. Mislivec, L. Aliaga et al., "The MINERvA Collaboration. Measurement of coherent production of $\pi^{ \pm}$in neutrino and antineutrino beams on carbon from $E_{v}$ of 1.5 to $20 \mathrm{GeV}$," Physical Review Letters, vol. 113, no. 26, Article ID 261802, 2014.

[5] M. Ahlers and F. Halzen, "High-energy cosmic neutrino puzzle: a review," Reports on Progress in Physics, vol. 78, no. 12, Article ID 126901, 2015.

[6] Y. Abe, J. C. dos Anjos, J. C. Barriere et al., "Improved measurements of the neutrino mixing angle $\theta_{13}$ with the Double Chooz detector," Journal of High Energy Physics, vol. 2014, no. 10, article 86, pp. 1-40, 2014.

[7] Y.-F. Li, J. Cao, Y. Wang, and L. Zhan, "Unambiguous determination of the neutrino mass hierarchy using reactor neutrinos," Physical Review D, vol. 88, no. 1, Article ID 013008, 9 pages, 2013. 
[8] J. Bergström, "Combining and comparing neutrinoless double beta decay experiments using different nuclei," Journal of High Energy Physics, vol. 2013, no. 2, pp. 1-26, 2013.

[9] P. Adamson, I. Anghel, C. Backhouse et al., "Measurement of neutrino and antineutrino oscillations using beam and atmospheric data in MINOS," Physical Review Letters, vol. 110, no. 25, Article ID 251801, 2013.

[10] T. Han, I. Lewis, R. Ruiz, and Z.-G. Si, "Lepton number violation and $W^{\prime}$ chiral couplings at the LHC," Physical Review D-Particles, Fields, Gravitation and Cosmology, vol. 87, no. 3, Article ID 035011, 2013.

[11] T. Asaka and T. Tsuyuki, "Seesaw mechanism at electronelectron colliders," Physical Review D, vol. 92, no. 9, Article ID 094012, 2015.

[12] M. Drewes, "The phenomenology of right handed neutrinos," International Journal of Modern Physics E, vol. 22, no. 9, Article ID 1392003, 2013.

[13] P. Ko and Y. Tang, "IceCube events from heavy DM decays through the right-handed neutrino portal," Physics Letters B, vol. 751, pp. 81-88, 2015.

[14] A. O. Barut and J. McEwan, "The four states of the massless neutrino with Pauli coupling by spin-gauge invariance," Letters in Mathematical Physics, vol. 11, no. 1, pp. 67-72, 1986.

[15] M. Drewes and B. Garbrecht, "Leptogenesis from a GeV seesaw without mass degeneracy," Journal of High Energy Physics, vol. 2013, article 96, 2013.

[16] M. Drewes, "Heavy neutrinos in particle physics and cosmology," in Proceedings of Science The European Physical Society Conference on High Energy Physics, vol. 2229 of PoS(EPS-HEP 2015)075, Vienna, Austria, July 2015.

[17] L. Canetti, M. Drewes, and B. Garbrecht, "Probing leptogenesis with GeV-scale sterile neutrinos at LHCb and Belle II," Physical Review D, vol. 90, no. 12, Article ID 125005, 9 pages, 2014.

[18] A. Palcu, "Neutrino mass as a consequence of the exact solution of 3-3-1 gauge models without exotic electric charges," Modern Physics Letters A, vol. 21, no. 15, pp. 1203-1217, 2006.

[19] S. M. Bilenky, "Neutrino," Physics of Particles and Nuclei, vol. 44, no. 1, pp. 1-46, 2013.

[20] E. Wigner, "On unitary representations of the inhomogeneous Lorentz group," Annals of Mathematics, vol. 40, no. 1, pp. 149204, 1939.

[21] Y. S. Kim and E. P. Wigner, "Cylindrical group and massless particles," Journal of Mathematical Physics, vol. 28, no. 5, pp. 1175-1179, 1987.

[22] P. A. Dirac, "Application of quaternions to Lorentz transformations," Proceedings of the Royal Irish Academy, vol. 50, pp. 261270, 1945

[23] M. A. Naimark, "Linear representation of the Lorentz group," Uspekhi Matematicheskikh Nauk, vol. 9, no. 62, pp. 19-93, 1954, Translated by F. V. Atkinson, American Mathematical Society Translations Series 2, vol. 6, pp. 379-458, 1957.

[24] Y. S. Kim and M. E. Noz, Theory and Applications of the Poincaré Group, Fundamental Theories of Physics, Kluwer Academic, Dordrecht, The Netherlands, 1986.

[25] S. Başkal, Y. S. Kim, and M. E. Noz, "Wigner's space-time symmetries based on the two-by-two matrices of the damped harmonic oscillators and the Poincaré sphere," Symmetry, vol. 6, no. 3, pp. 473-515, 2014.

[26] V. B. Berestetskii, E. M. Lifshit, and L. P. Pitaevskii, Quantum Electrodynamics, vol. 4 of The Course of Theoretical Physics, Pergamon Press, Oxford, UK, 2nd edition, 1982.
[27] S. Bakal, Y. S. Kim, and M. E. Noz, Physics of the Lorentz Group, Morgan \& Claypool Publishers IOPScience, Bristol, UK, 2015.

[28] V. Bargmann, "Irreducible unitary representations of the Lorentz group," Annals of Mathematics, vol. 48, pp. 568-640, 1947.

[29] S. Başkal and Y. S. Kim, "de Sitter group as a symmetry for optical decoherence," Journal of Physics A: Mathematical and General, vol. 39, no. 24, 2006.

[30] D. Han, Y. S. Kim, and D. Son, "E(2)-like little group for massless particles and neutrino polarization as a consequence of gauge invariance," Physical Review D, vol. 26, no. 12, pp. 3717-3725, 1982.

[31] D. Han, Y. S. Kim, and D. Son, "Photon spin as a rotation in gauge space," Physical Review D, vol. 25, no. 2, pp. 461-463, 1982.

[32] D. Han, M. E. Noz, Y. S. Kim, and D. Son, "Space-time symmetries of confined quarks," Physical Review D, vol. 25, no. 6, pp. 1740-1743, 1982.

[33] D. Han, Y. S. Kim, and D. Son, "Photons, neutrinos, and gauge transformations," American Journal of Physics, vol. 54, pp. 818821, 1986

[34] D. Han, Y. S. Kim, and D. Son, "Eulerian parametrization of Wigner's little groups and gauge transformations in terms of rotations in two-component spinors," Journal of Mathematical Physics, vol. 27, no. 9, pp. 2228-2235, 1986.

[35] Y. S. Kim, "Neutrino polarization as a consequence of gauge invariance," Czechoslovak Journal of Physics, vol. 52, no. S3, pp. C353-C360, 2002.

[36] Y. S. Kim and M. E. Noz, "Symmetries shared by the Poincaré Group and the Poincaré Sphere," Symmetry, vol. 5, no. 3, pp. 233-252, 2013. 

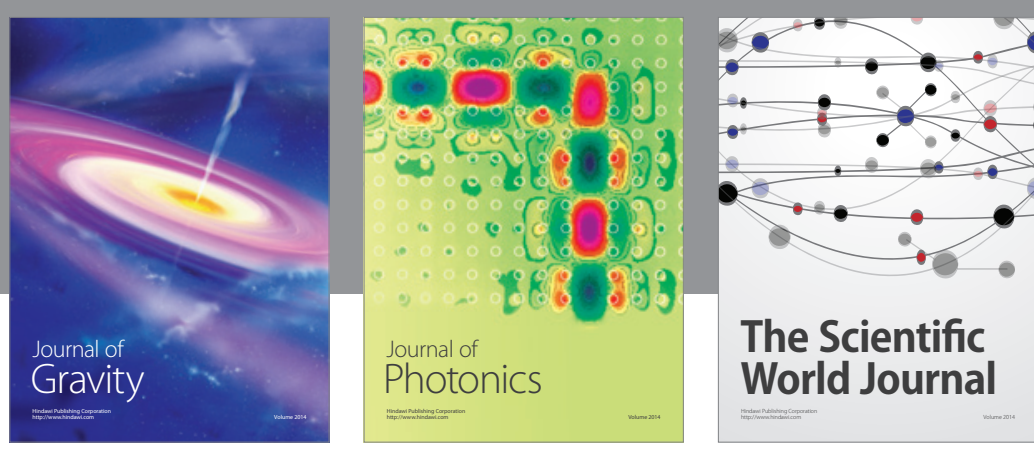

The Scientific World Journal
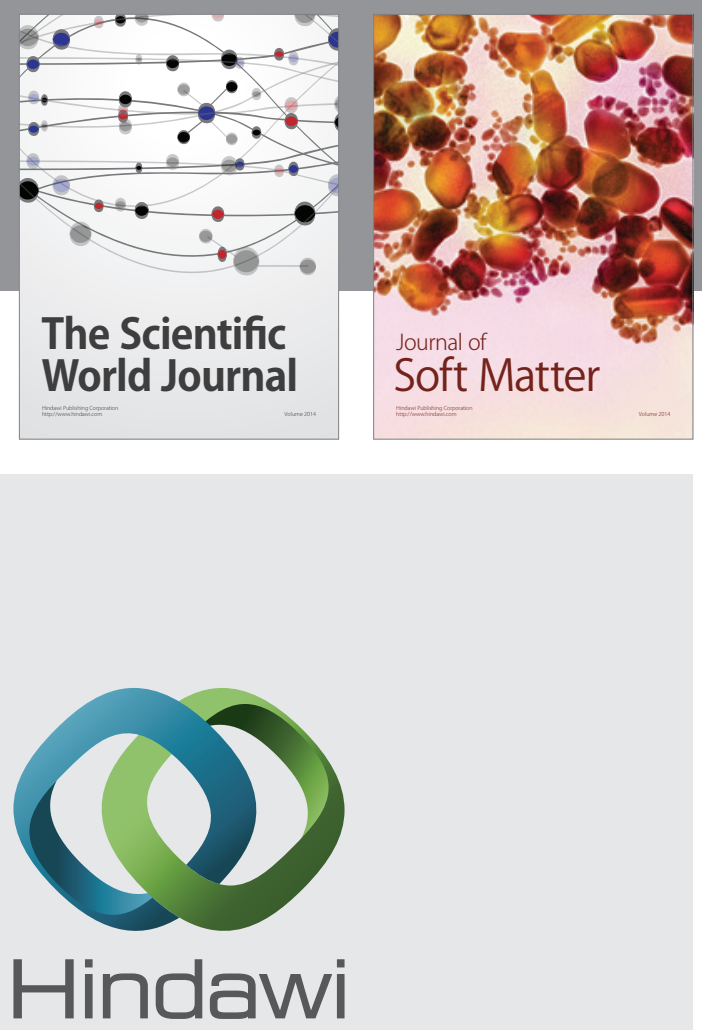

Submit your manuscripts at

http://www.hindawi.com

nternational Journal of

Statistical Mechanics
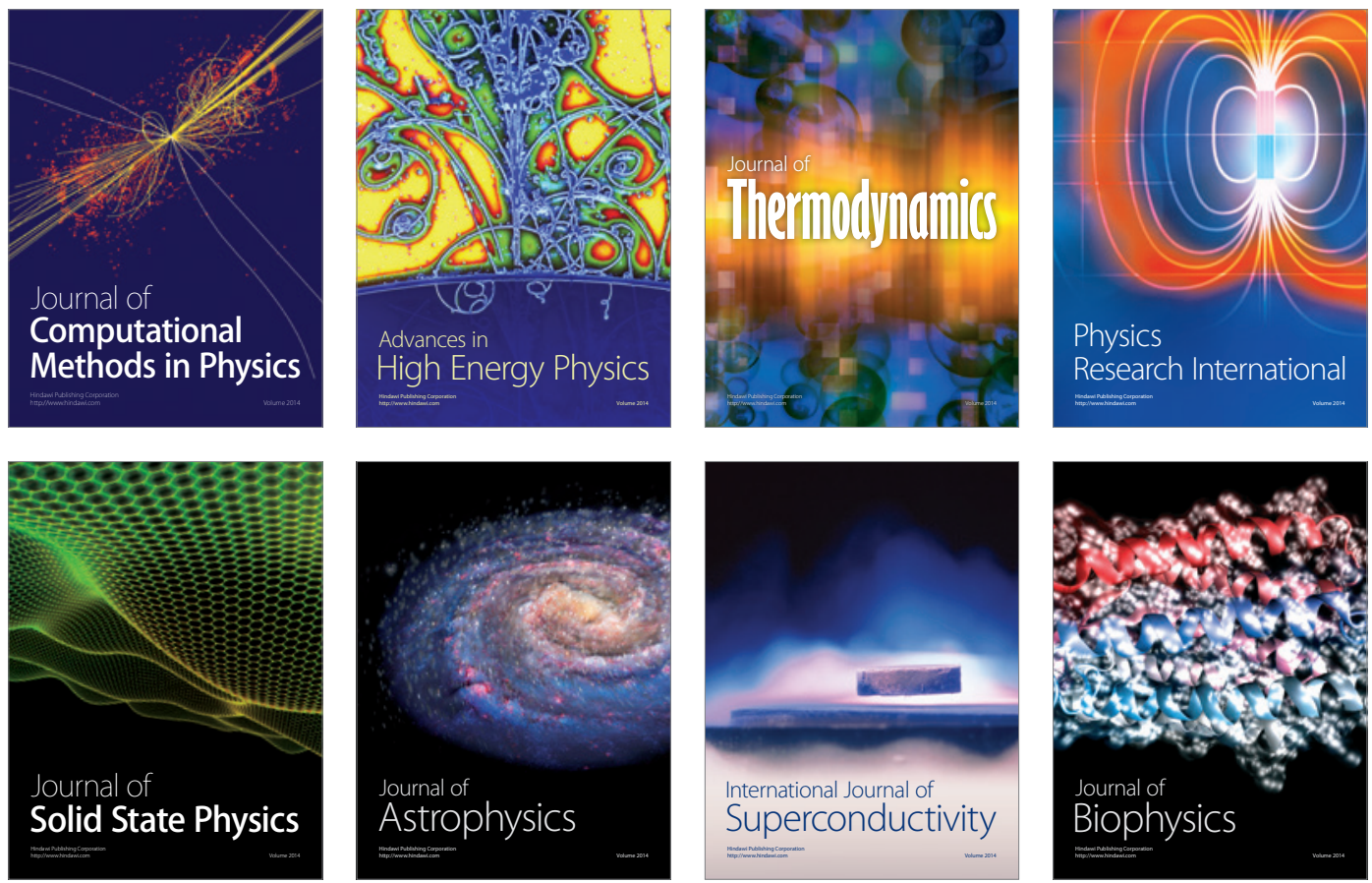
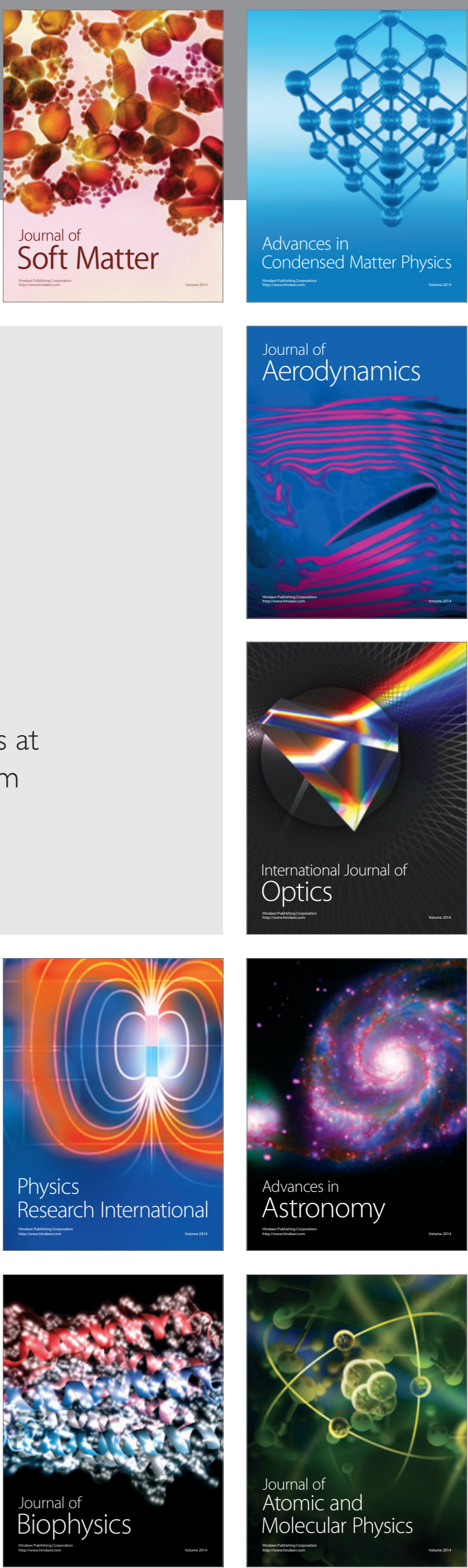